EPJ Web of Conferences 75, 02004 (2014)

DOI: $10.1051 /$ epjconf/ 20147502004

(C) Owned by the authors, published by EDP Sciences, 2014

\title{
Transient magnetic tunneling mediated by a molecular bridge in the junction region
}

\author{
A. Kalvoválab, V. Špička ${ }^{1}$, and B. Velický2 \\ 1 Institute of Physics ASCR, Na Slovance 2, 18221 Praha 8, Czech Republic \\ 2 Dept. of Condensed Matter Physics, Charles University, 12116 Praha 2, Czech Republic
}

\begin{abstract}
This paper extends our recent theoretical study of transient currents in molecular bridge junctions [1] to magnetic tunneling. Presently, we calculate the excess magnetic tunneling through the molecular bridge shunting the junction. The system is represented by two ferromagnetic electrodes bridged by a molecular size island with one electronic level and a local Hubbard type correlation. The island is linked with the electrodes by tunneling junctions whose coupling strength is assumed to undergo rapid changes affecting the connectivity of the system. We employ the non-equilibrium Green's functions. The numerical solution is obtained solving the real-time Dyson equation in the integro-differential form self-consistently. The switching events controlling the junctions give rise to transient changes of magnetisation of the island. They strongly depend on the static galvanic bias between the electrodes, mutual alignment of their magnetisation and on the time scale of the switching.
\end{abstract}

\section{Introduction}

In this paper, we study numerically transient electronic processes induced by rapid switching in a model nanoscopic structure, a single level molecular bridge sandwiched between two ferromagnetic leads by independent tunneling junctions. This extends our previous study of such rapid time dependent processes in a similar stucture with leads consisting of simple metals [1].

The molecular bridge structure has served as a prototype nanoscopic system for an extensive study from various angles as reviewed in [2]. Many recent studies aim at a high level of sophistication of the model: a systematic account of many-electron interactions based on the modern timedependent density functional theory [3], inclusion of phonon reservoirs see e.g. [4]. Our work belongs to the less investigated question of the fast transient processes [5]. These processes may be induced externally (light pulses) or internally, in particular by rapid changes of the coupling strength of the junctions. We concentrate on the latter mechanism. The basic formal tool are naturally the non-equilibrium Green's functions. The two fundamental papers are [6] for stationary currents and in particular Ref. [7] for time-dependent phenomena. In paper [8], a detailed formal framework for our approach to the transient processes in such nanoscopic structures is given. Two question we have addressed are the formal tools for incorporating the correlated initial conditions for transients starting at a finite initial time, and the gradual transition from the correlated early stage of the transient to a kinetic regime.

These formal questions were analyzed numerically in [1] with the emphasis on the whole chain of switching events. The extension to the spin polarized processes in the present paper is devoted to just a pair of fast switch-on

\footnotetext{
a e-mail: kalvova@fzu.cz

b This research was supported by the Czech Science Foundation within the grant project P204/12/0897
}

events, but the role of the galvanic bias and of the strength of the local Coulomb interaction in the island on its transient local magnetic moment is studied in detail.

\section{Model and Formalism}

The model Hamiltonian is a straightforward extension of the Hamiltonian used previously in [1], [8], [9] and belongs to the family of Hamiltonians introduced in [7] :

$$
\begin{aligned}
\hat{H}(t) & =\hat{H}_{0}+\hat{H}^{\prime}(t) \\
\hat{H}_{0}(t) & =\hat{H}_{0 L}+\hat{H}_{0 B}+\hat{H}_{0 L} \\
\hat{H}_{0 B}(t) & =\varepsilon_{b}\left(\hat{n}_{b \uparrow}+\hat{n}_{b \downarrow}\right)+U \hat{n}_{b \uparrow} \hat{n}_{b \downarrow} \\
\hat{H}^{\prime}(t) & =\alpha_{L} V_{L}+\alpha_{R} V_{R}
\end{aligned}
$$

We assume a strictly collinear geometry, so that the one-electron part of the Hamiltonian is spin-diagonal. In particular, the leads are assumed to have identical electron structures with the up-spin and down-spin bands mutually shifted by a constant exchange splitting. The island-lead coupling amplitudes are taken as spin independent and corresponding to the ideal junctions. All time dependence of the Hamiltonian is contained in the scalar functions describing a loosening of the respective junction. Simple onand-off switching is described by a function varying stepwise between 0 and 1 . The island has a single level with Coulomb interaction. The interaction term is the only one responsible for the mutual coupling of electrons with opposite spin in the whole Hamiltonian. The Hubbard $U$ is considered as weak enough to permit the use of the meanfield approximation. The spin coupling is then time-local and its role in the evolution of the transients is thus easier to be followed.

While we will consider transients starting at a finite intial time, the convenient general framework for introducing the 
NGF is to follow the Keldysh scheme [10] employing an adiabatic process starting from $\hat{H}_{0}$ at $t \rightarrow-\infty$. Similarly as in [7], it is easy to obtain the Keldysh NGF for the central island orbital. We employ the Langreth-Wilkins convention [11] and work with the triplet of propagators $G^{R}, G^{A}$ and the "less" particle correlation function $G^{<}$of Kadanoff and Baym [12]. The Dyson equations for the propagators read

$$
G_{\sigma}^{R, A}=G_{0 \sigma}^{R, A}+G_{0 \sigma}^{R, A} \sum_{\sigma}^{R, A} G_{\sigma}^{R, A}, \quad \sigma=\uparrow, \downarrow
$$

while the less component satisfies the Dyson equation

$$
G_{\sigma}^{<}=G_{\sigma}^{R} \Sigma_{\sigma}^{<} G_{\sigma}^{A}, \quad \sigma=\uparrow, \downarrow
$$

All components of the self-energy have the form

$$
\begin{aligned}
\sum_{\sigma}^{X}\left(t, t^{\prime}\right) & =\Sigma_{L \sigma}^{X}\left(t, t^{\prime}\right)+\mathrm{i} G_{\bar{\sigma}}^{<}(t, t) \delta\left(t-t^{\prime}\right)+\sum_{R \sigma}^{X}\left(t, t^{\prime}\right) \\
\sum_{Y \sigma}^{X}\left(t, t^{\prime}\right) & =\alpha_{Y}(t) \tilde{\Sigma}_{Y \sigma}^{X}(\tau) \alpha_{Y}\left(t^{\prime}\right) \\
\tilde{\Sigma}_{Y \sigma}^{R, A}(\tau) & = \pm \mathrm{i} \int \frac{d E}{2 \pi} \Delta_{Y \sigma}(E) \mathrm{e}^{-\mathrm{i} E \tau} \vartheta( \pm \tau) \\
\Sigma_{Y \sigma}^{<}(\tau) & =\mathrm{i} \int \frac{d E}{2 \pi} \Delta_{Y \sigma}(E) f_{Y}(E) \mathrm{e}^{-\mathrm{i} E \tau} \\
\tau & =t-t^{\prime}, \quad X=R, A,<\quad Y=L, R
\end{aligned}
$$

Each fully connected junction is thus associated with a pair of spectral functions $\Delta_{Y \sigma}$ which, together with the quasiequilibrium Fermi function $f_{Y}$, specify the Y self-energy triplet.

In principle, any time-dependent process at the bridge is now solvable. We are specifically interested in transients suddenly starting at a finite time. Such processes may be conveniently viewed as embedded into the complete Keldysh "host" process.

This does not affect the propagators, but the Dyson equation for the embedded less function becomes more involved, as it incorporates the initial condition and a memory of the previous evolution history. A general formalism for this is available, the so-called partitioning in time [13]. The simplest process considered in this paper starts from the initial state, in which the island is completely disconnected from the leads up to the initial time $t_{0}$ This corresponds to an uncorrelated initial condition, for which the time partitioned formalism simplifies to the often quoted so-called Keldysh initial condition. We indicate here the corresponding Dyson equation for $G^{<}$in the form of an integro-differential equation:

$$
\begin{aligned}
& \mathrm{i} \partial_{t} G_{\sigma}^{<}\left(t, t^{\prime}\right)=\left(\varepsilon_{b}-\mathrm{i} U G_{\bar{\sigma}}(t, t)\right) G_{\sigma}^{<}+\int_{t_{0}}^{t} \Sigma_{\sigma}^{R} G_{\sigma}^{<}+\int_{t_{0}}^{t^{\prime}} \Sigma_{\sigma}^{<} G_{\sigma}^{A} \\
& G_{\sigma}^{<}\left(t=t_{0}, t^{\prime}\right)=\rho_{0} \cdot G_{\sigma}^{A}\left(t_{0}, t^{\prime}\right)
\end{aligned}
$$

The initial condition is specified in the second line. It is apparent that $G^{<}$is coupled with the propagators which satisfy similar equations, and the whole system is solved self-consistently as an incremental time process.

\section{Transient magnetisation as a function of external bias}

Even our oversimplified model still depends on an extensive parameter input. In Fig.1, an overview of our actual choice is shown. In equilibrium, the leads have equilibrated

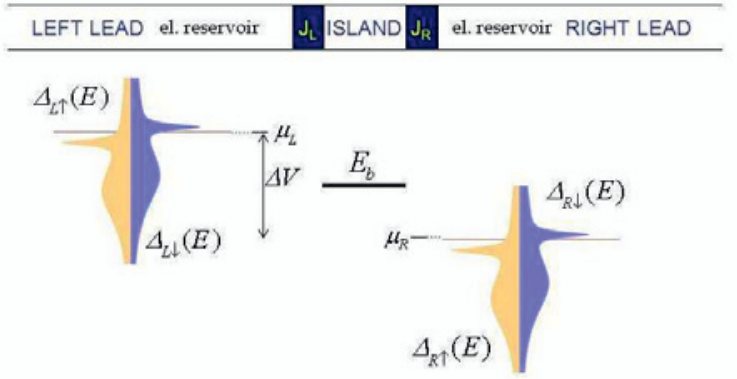

Fig. 1. Sketch of the molecular bridge. The leads are given a symmetrical bias with respect to the empty level of the island bound state as given by the difference of the quasi-Fermi levels, . The tunneling spectral densities float with the respective Fermi levels. Shown is the case of a parallel magnetization of both leads.

Fermi levels and the island bound state is empty and floating just above. The tunnelling spectral functions are pinned to their Fermi levels. As shown in the figure, the two leads are then given a symmetrical bias with respect to the island level.

In the studies of molecular bridges, the spectral function is customarily taken as flat (the so-called wide band limit). In fact, this function is physically very similar to the adsorption function of Grimley or Newns [14] and may have a pronounced spectral structure reflecting the density of states in the lead. In the present case of itinerant ferromagnets, it is reasonable to model, in a simple manner, the sp-d structure of the density of states. Our choice is sketched in the figure, from which also the magnitude of the exchange splitting is apparent. As an overview of the dynamical behaviour of this model we present the up and down spin occupancy of the island level during the first 50 fs after the left lead has been suddenly attached. In Fig. 2 , this evolution is plotted for a number of bias voltages. The plots form together the darker surface in the plot. For clarity, every tenth curve is heavier. The following features emerge: For all biases in the range, the occupancy starts with rapid coherent oscillations which gradually decay and are followed by a kinetic stage, during which the occupancies relax to their saturation values. The behaviour of the up spin electrons and the down spin electrons is radically different and this results into the non-zero magnetization of the island plotted in the third figure. Remarkably, the dependence of the occupancy numbers on the bias displays a sudden change of the asymptotic time behaviour at about $0.5 \mathrm{eV}$. This value of the critical bias depends on the other parameters. We will return to this in the following section.

The lighter surfaces splitting off in the plots of Fig. 2 at 25 fs correspond to connecting also the right lead. This onset time of the second transient is chosen, because the first transient is still far from saturation, but the initial coherent oscillations have largely decayed off. The evolution of the second transient resembles the first one in gross features, but there are pronounced differences in details, because the second transient starts from a correlated initial condition note the irregular dependence of the early oscillations on the bias. 


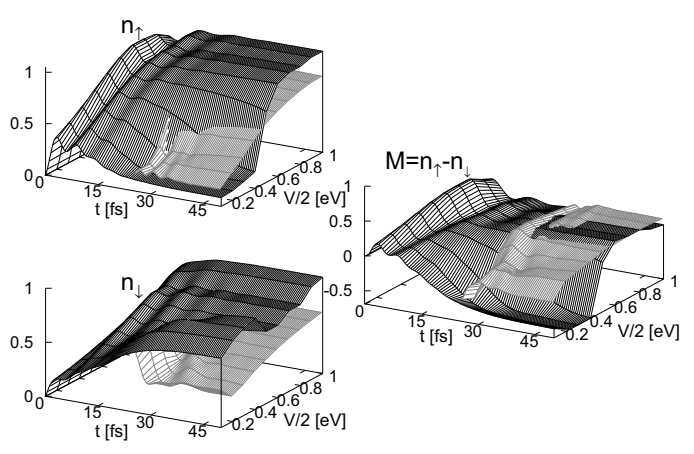

Fig. 2. Island level occupancies and island magnetization (their difference) as a function of time and the bias. Darker surface: At $t_{0}=0$ the left lead is suddenly attached. Lighter surface: at $t=25 \mathrm{fs}$ the right lead is additionally attached. $U=0.6 \mathrm{eV}$, the bias voltage $V$ is varied and serves as the second axis of all plots.

\section{Analysis of the results. Role of the Hubbard U}

In the preceding section, the $\mathrm{t}-\mathrm{V}$ plots were shown first because of their experimental relevance. It was not easy to analyse them, because changing the bias means also to change the position of the Fermi levels. Here, we will make a more convenient complementary choice: keep the bias fixed and make the Hubbard U variable.

In Fig. 3, the plots shown in a format similar to Fig. 2 , but with $U$ as the second variable, manifest also two regimes. It is remarkable that the majority up spin is little sensitive to the $U$ value, while the opposite spin direction undergoes a jump at about $\mathrm{U}=0.6 \mathrm{eV}$ which gives rise to a pronounced two mode behaviour of the saturation magnetization. This "long time" behaviour is in clear contrast to the early transient stage, during which the rise of the occupancies is nearly $U$ independent.

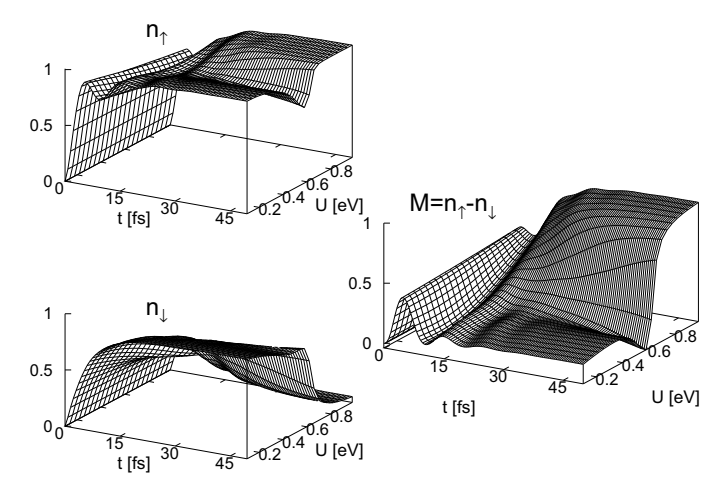

Fig. 3. Island level occupancies and island magnetization as a function of time and of the Coulomb integral (Hubbard U). Only the left lead is turned on.

Fig. 3 can be understood in terms of the time dependence of the opposite spin direction. Considering first the very short time behaviour, the occupancy of the level is only beginning to rise and the same holds for the renormalized levels, thus the role of $U$ is limited, in agreement with the plots. Later, however, the level shifts become significant and this induces changes in their occupancies. The process is self-consistent. To get an insight into its mech- anism, we plot the spectral densities of the island level at the time of the asymptotic saturation. They are shown in Fig. 4, again in their parametric dependence on U. In red, the plane corresponding to the Fermi level of the left lead is drawn. To the left of the plane, the spectral density corresponds to occupied states, to the right, to the empty ones. Both spectral densities have a two peak character, but the up spin density remains occupied throughout, while the down spin level emerges from the Fermi sea, its occupancy decreases and this makes the up spin spectral density similar for the small and the large U.
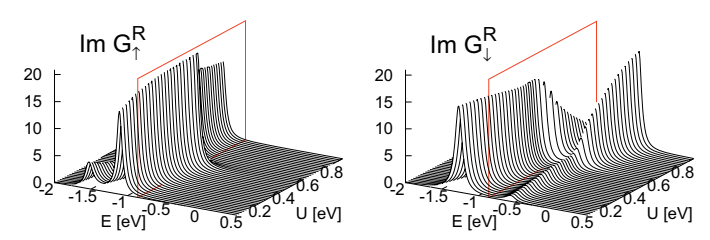

Fig. 4. The asymptotic spectral density of the island level for both spin oprientations as a function of energy and the Hubbard $U$.

With this basic picture of the first transient, we may turn on also the second lead, after elapsing of $25 \mathrm{fs}$ again. The result of the calculation is shown in Fig. 5. In this figure, the dark surface is identical with the plots of Fig. 3 and serves as a reference. Two additional surfaces split off at 25 fs. The light grey surfaces correspond to the parallel magnetization of both leads, the blue ones to the anti-parallel magnetization. It is remarkable that " the magnetization at the island follows the sign of magnetization of the lead which serves as a drain; " the magnetization for the antiparallel arrangement undergoes a jump at a critical U.

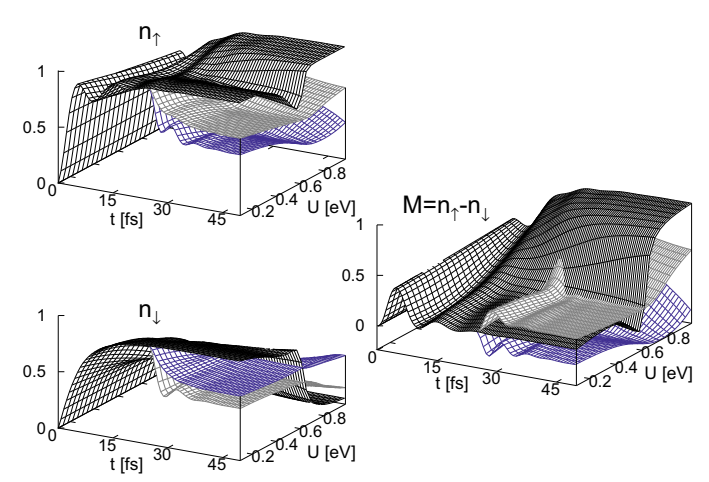

Fig. 5. . Island level occupancies and island magnetization as a function of time and of the Coulomb integral (Hubbard U). Dark grey surface: Only the left lead is turned on (cf. Fig. 3). Light grey surface: Right lead turned on at $25 \mathrm{fs}$, parallel magnetization of the leads. Blue surface: Right lead turned on at $25 \mathrm{fs}$, anti-parallel magnetization of the leads.

\section{References}

1. A. Kalvová, V. Špička, B. Velický, J. Supercon. and Novel Mag. 26, 773 (2013)

2. M. Galperin, M. A. Ratner, and A. Nitzan, J. Phys.: Cond. Matt. 19, 103201 (2007) 
3. G. Stefanucci, Phys. Rev. B75, 195115 (2007)

4. D.F. Urban, R. Avriller, A. Levy Yeyati, Phys. Rev. B82, 121414(R) (2010)

5. 5. T.L. Schmidt, P. Werner, L. Muhlbacher, A. Komnik, Phys. Rev. B78, 235110 (2008)

6. Y. Meir, N.S. Wingreen, Phys. Rev. Lett. 68, 2512 (1992)

7. A. P. Jauho, N.S. Wingreen, Y. Meir, Phys. Rev. B 50, 5528 (1994)

8. V. Špička, A. Kalvová, B. Velický, Phys. Scr. T151, 014037 (2012)

9. A. Kalvová, V. Špička, B. Velický, Non-equilibrium Statistical Physics Today, AIP Conf. Proc. 1332, 223 (2011)

10. 10. L.V. Keldysh, Sov. Phys. JETP 20, 1018 (1965)

11. L.P. Kadanoff, G. Baym, Quantum Statistical Mechanics, Benjamin, New York, 1962

12. B. Velický, A. Kalvová, V. Špička,Phys. Rev. B 81, 235116 (2010)

13. D.M. Newns, Phys. Rev. 178, 1123 (1969);

T.B.Grimley, J. Vac. Sci. Technol. 8, 31 (1971) 